\title{
In-Situ Transmission Electron Microscopy: Electron Beam Effects in Carbon-based Nanomaterials
}

Zhehan Ying ${ }^{1}$, Jiangyong Diao ${ }^{2}$, Shi Wang $^{3}$, Xiangbin Cai $^{3}$, Hongyang Liu $^{2}$ and Ning Wang ${ }^{1}$

${ }^{1}$ The Hong Kong University of Science and Technology, Hong Kong, Hong Kong, ${ }^{2}$ Institute of Metal Research, Chinese Academy of Sciences, United States, ${ }^{3}$ The Hong Kong University of Science and Technology, Hong Kong

Carbon-based nanomaterials, such as onion-like carbon (OLC), carbon nanotube (CNT) and graphene oxide (GO) are highlighted in catalysis and engineering fields by virtue of their outstanding physical and chemical properties including thermal stability, mechanical strength, corrosion resistance and structural diversity. ${ }^{[1-4]}$ More recently, the in-situ transmission electron microscopy (TEM) has been widely applied for solving challenges in materials science such as investigating the thermal stability of nanomaterials, revealing the deactivation mechanism of nanomaterials and determining the synthetic routes of nanomaterials, which is conducive to connecting the relationships among property, structure and synthesis of nanomaterials. ${ }^{[5-7]}$ It is worthwhile mentioning that spurious effects caused by the electron beam should be taken into account when conducting in-situ TEM investigations of the thermal stability or electrochemical characteristics of carbon-based nanomaterials. The electron beam would potentially introduce the excess heat on the specimen, free radicals, knock-on damage and surface sputtering. ${ }^{[8]}$ In general, the scientists capture an image quickly to minimize the influences of the electron beam on target areas, however, the impacts on some sensitive nanomaterials remain. The optimal approach is to give consideration to both TEM imaging quality and electron beam effects, and thus observe the sample under certain threshold values of electron beam flux to avoid the structural transformation of carbon-based nanomaterials. In the following, we focus on electron beam effects in the carbon-based nanomaterials mentioned above and then provide feasible threshold values of electron beam flux for OLC, CNT and GO for the future in-situ TEM studies.

The in-situ TEM experiments were operated on MEMS-based E-chips loaded on a Protochips Fusion Select double-tilt holder, allowing thermal or electrical in-situ observations on the sample. The electronbeam-induced microstructural changes of carbon-based nanomaterials were investigated by a JOEL 2010 FEG (field emission gun) transmission electron microscopy microscope operating at $200 \mathrm{kV}$ (point-topoint resolution: $1.2 \AA) .{ }^{[9]}$ The morphology images of palladium nanoparticles deposited on the OLC (PdNPs/OLC), palladium nanoparticles deposited on the CNT (PdNPs/CNT) and platinum nanoparticles deposited on the GO (PtNPs/GO) captured at different magnifications levels are displayed in Figure 1. The metal nanoparticles are evenly dispersed on their surfaces. The OLCs (Figure 1a) consist of multiple fullerene-like enclosed carbon shells and appear as spherical shape; the CNTs (Figure 1b) consist of multiple rolled layers of graphene and appear as cylindrical nanostructures; the GOs (Figure 1c) are chemically-modified graphene consisting a single-atomic layered graphite with various oxygencontaining functionalities and exhibit smooth surfaces. In-situ time-resolved TEM image series (Figure 2) was recorded continuously to demonstrate electron beam effects in the OLC, CNT and GO nanomaterials under different electron beam fluxes. Under the relatively high electron beam flux (current density $=15 \mathrm{pA} / \mathrm{cm}^{2}$ ) for 20 mins, the microstructural destruction of OLC and CNT was clearly observed. The spherical carbon-shell structures of OLCs experienced evident changes after 10 mins, and thorough destruction after 20 mins irradiation (Figure 2a-c). The multiple carbon layers of CNTs deformed after 10 mins, and subsequently shrank and necked after 20 mins irradiation (Figure 2g-i). In comparison, GO can resist the electron beam flux with current density up to $20 \mathrm{pA} / \mathrm{cm}^{2}$, which satisfies most of the 
irradiation conditions for TEM experiments. (Figure 2m-o). To diminish electron beam effects, in-situ thermal or electrical studies should be performed under electron beam flux below a threshold value of current density. Through several trials of current density gradients on the three carbon-based nanomaterials, it was determined that the microstructures of OLC (Figure 2d-f) and CNT (Figure j-l) would not change under electron beam flux with the current density of $5 \mathrm{pA} / \mathrm{cm}^{2}$ and $7 \mathrm{pA} / \mathrm{cm}^{2}$, respectively. The aforementioned results reveal the observation of electron-beam-induced microstructural changes and validate threshold values of electron beam effects of OLC, CNT and GO nanomaterials, which are conducive to their in-situ thermal or electrical TEM studies afterwards.
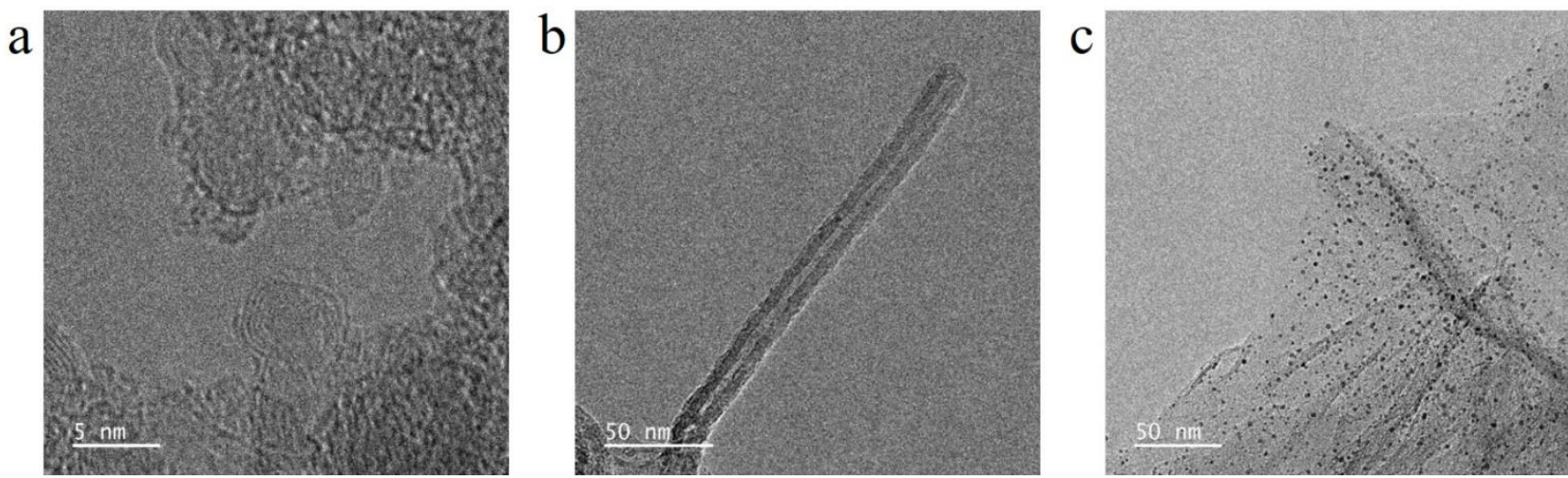

Figure 1.TEM morphology images of (a) PdNPs/OLC Magnification (MAG) $\times 800 k$; (b) PdNPs/CNT, MAG $\times 100 \mathrm{k}$; (c) PtNPs/GO, MAG $\times 80 \mathrm{k}$. 

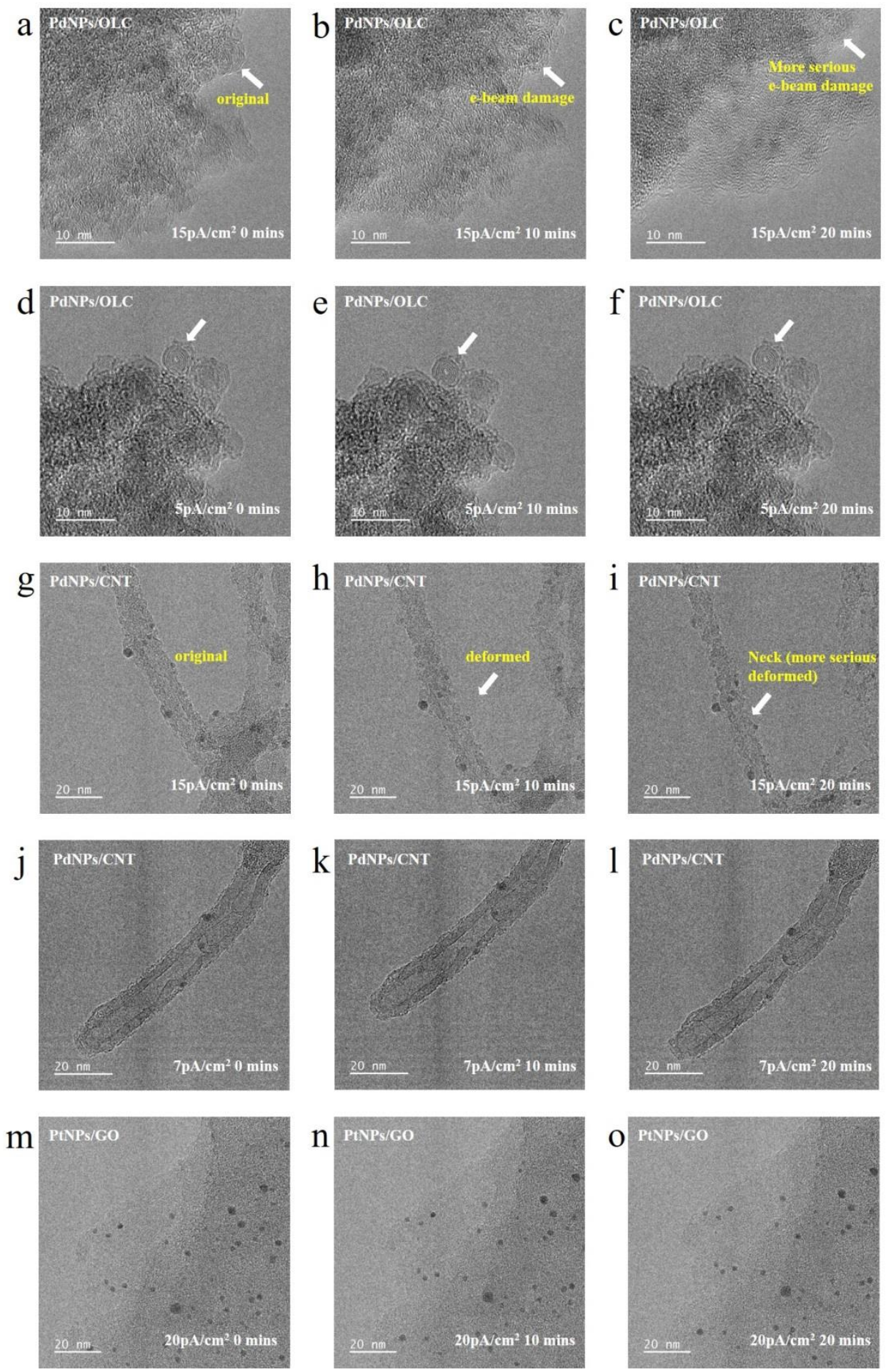

Figure 2. In-situ TEM time-resolved image series of (a-c) PdNPs/OLC under $15 \mathrm{pA} / \mathrm{cm} 2$ current density for 0 mins, 10 mins and 20 mins (In convenience of your comparison, white arrows are used to indicate the target OLC); (d-f) PdNPs/OLC under $5 \mathrm{pA} / \mathrm{cm} 2$ current density for 0 mins, 10 mins and 20 mins; (g-i) PdNPs/CNT under 15 $\mathrm{pA} / \mathrm{cm} 2$ current density for 0 mins, 10 mins and 20 mins; (j-1) PdNPs/CNT under $7 \mathrm{pA} / \mathrm{cm} 2$ current density for 0 mins, 10 mins and 20 mins; (m-o) PtNPs/GO under 20 pA/cm2 current density for 0 mins, 10 mins and 20 mins.

\section{References}


[1] Y. Yan, J. Miao, Z. Yang, F.-X. Xiao, H. B. Yang, B. Liu, Y. Yang, Chemical Society Reviews, 2015, 44, 3295.

[2] H. Liu, L. Zhang, N. Wang, D. S. Su, Angewandte Chemie International Edition, 2014, 53, 12634.

[3] M. Zeiger, N. Jäckel, V. N. Mochalin, V. Presser, Journal of Materials Chemistry A, 2016, 4, 3172.

[4] C. Su, M. Acik, K. Takai, J. Lu, S.-j. Hao, Y. Zheng, P. Wu, Q. Bao, T. Enoki, Y. J. Chabal, Nature communications, 2012, 3, 1.

[5] A. T. DeLaRiva, T. W. Hansen, S. R. Challa, A. K. Datye, Journal of catalysis, 2013, 308, 291.

[6] B. He, Y. Zhang, X. Liu, L. Chen, ChemCatChem, 2020.

[7] M. Tang, W. Yuan, Y. Ou, G. Li, R. You, S. Li, H. Yang, Z. Zhang, Y. Wang, ACS Catalysis, 2020, $10,14419$.

[8] R. Egerton, P. Li, M. Malac, Micron, 2004, 35, 399.

[9] We acknowledge the technical support from Materials Characterization and Preparation Facility of The Hong Kong University of Science and Technology and financial assistance from Research Grants Council of Hong Kong (Project No. 16306818 and N_HKUST624/19), National Key R\&D Program of China (2016YFA0204100), National Natural Science Foundation of China (91845201, 21961160722, 22072162), Liaoning Revitalization Talents Program (XLYC1907055), Guangxi Key Laboratory of Information Materials Guilin University of Electronic Technology (Project 191005-K) and the Sinopec China. 